\title{
Percepción de la conducta de los padres en los comportamientos antisociales mostrados por los jóvenes participantes de deportes colectivos*
}

\author{
Perception of Parents' Conducts on Antisocial Behaviors \\ Shown by Team Sports Youth Athletes
}

Recibido: septiembre 12 de 2012 | Revisado: marzo 19 de 2013 | Aceptado: mayo 9 de 2013

\author{
Pedro Antonio Sánchez Miguel ** \\ JuAN José Pulido GONZÁLEZ *** \\ Diana AMADO AlONSO \\ DAVID SÁNCHEz OLIVA \\ Francisco Miguel Leo Marcos \\ Universidad de Extremadura, España
}

doi:10.11144/Javeriana.UPSY13-1.pcpc

Para citar este artículo: Sánchez, P. A., Pulido, J. J., Amado, D., Sánchez, D. \& Leo, F. M. (2014). Percepción de la conducta de los padres en los comportamientos antisociales mostrados por los jóvenes participantes de deportes colectivos. Universitas Psychologica, 13 (1), 299-309. doi:10.11144/ Javeriana.UPSY13-1.pcpc

* Artículo de investigación.

** Profesor ayudante Departamento de Didáctica de la expresión musical, plástica y corporal. Universidad de Extremadura, España. E-mail: pesanchezm@unex.es

**** E-mails: jpuligog@unex.es, diamal@unex.es, davidsanchez@unex.es,franmilema@unex.es

\section{RESUMEN}

El principal objetivo del estudio fue conocer las relaciones entre padres e hijos en la percepción de intención, actuación y juicio de los posibles comportamientos antisociales en el contexto deportivo, valorando en una misma acción deportiva, la moralidad mostrada por ambos significativos y considerando el clima fomentado por los padres en los deportistas para la aparición o no de estas conductas. La muestra estuvo compuesta por 1.420 participantes, 710 deportistas, con edades comprendidas entre los 11 y 16 años $(M=12.76 ; \mathrm{DE}=1.15)$ y 710 padres, con edades comprendidas entre los 36 y 49 años $(M=43.56$; $\mathrm{DE}=2.95)$, de varios deportes colectivos: fútbol, baloncesto, balonmano y voleibol. Deportistas y padres diligenciaron cuestionarios destinados a valorar la percepción de los jugadores y padres sobre comportamientos antisociales y la percepción del clima motivacional que los padres transmiten a los deportistas. Los resultados muestran una relación significativa entre las variables antisociales en padres e hijos. Asimismo, la presión de los padres emerge como predictor de la intención y actuación de comportamientos antisociales. Finalmente, se indica que una participación adecuada de los padres en la práctica deportiva, supondrá el descenso de comportamientos antisociales por parte de los deportistas. Palabras clave autores

Moralidad, deporte, comportamientos antisociales, clima motivacional.

Palabras clave descriptores

Percepción, psicología del deporte, investigación cuantitativa.

\section{A B S T R ACT}

The main aim of the study was to know the relationships between parents and children with regard to perception of intention, performance and judgment of antisocial behaviors in the sport context, assessing in the same sport action, the morality shown by both significatives. The sample comprised 1420 participants, 710 athletes, ranging in age from 11 to 16 years old $(\mathrm{M}=12.76 ; \mathrm{SD}=1.15)$, and 710 parents, ranging in age from 36 to 49 years old $(\mathrm{M}=43.56 ; \mathrm{SD}=2.95)$. Athletes and parents completed questionnaires related to measure perception of players and parents' about antisocial behaviors, as well as perception of motivational climate developed by parents. Results showed a significant relationship among antisocial variables in parents and children. Moreover, parents' pressure emerged as predictor of intention and performance of antisocial behaviors. Finally, it is 
emphasized that appropriate parental participation in sport practice can promote the decrease of antisocial behaviors in athletes.

Key words authors

Morality, team sports, antisocial behaviors, motivation.

Key words plus

Perception, sport psychology, quantitative research.

\section{Introducción}

Asiduamente, en el deporte se producen situaciones que ponen en entredicho el "juego limpio" de los jugadores (Abenza, Bravo \& Olmedilla, 2006). Este tipo de acciones antideportivas se reproducen en categorías inferiores, siendo este un colectivo en el que se tienen que unir todos los esfuerzos para erradicarlas (Leo, Sánchez-Miguel, Sánchez-Oliva, Gómez \& García-Calvo, 2009). Además, las etapas de formación se convierten en un escenario propicio en el que se ponen de manifiesto situaciones que sirven para despertar el espíritu crítico de los jóvenes deportistas (Kavussanu, 2007).

Actualmente, las administraciones encargadas de fomentar el deporte de iniciación centran sus esfuerzos para que exista una finalidad más allá de la propia competición, sirviendo este contexto para el desarrollo de valores sociales (Jowett \& Timson-Katchis, 2005; Vallerand, 2007), basándose no solo en los participantes, sino aunando sus esfuerzos en un trabajo conjunto de los significativos del deporte, como son el propio deportista, los entrenadores, padres y árbitros (García-Calvo, Sánchez-Oliva, Sánchez-Miguel, Leo \& Amado, 2012; Sánchez-Miguel, Leo, Sánchez-Oliva, Amado \& García-Calvo, en prensa). Estas posibilidades no hacen mención exclusiva a las conocidas en el ámbito cognitivo, motor y expresivo, sino que también realzan la función social de la actividad física y el deporte (Lee, Whitehead \& Balchin, 2000; Lee, Whitehead, Ntoumanis \& Hatzigerorgiadis, 2008) a los que se ha catalogado como un excelente vehículo para el desarrollo de valores y demás aspectos morales (Kavussanu \& Boardley, 2009; Luckwü \& Guzmán, 2011; Sánchez-Oliva, Leo, SánchezMiguel, Amado \& García-Calvo, 2012), donde la competición deportiva se considera determinante para los comportamientos de los participantes (Kavussanu, Seal \& Phillips, 2006).

De esta manera, cuando el deporte se enfoca desde una perspectiva educativa, contribuye decisivamente a la promoción de la moralidad, ya que se encuentra vinculado a todas las esferas de la vida, desempeñando un papel muy importante en la conducta humana (Sage, 2006). En relación con ese tópico, se define comportamiento antisocial como una dimensión que hace referencia a las conductas dirigidas a hacer daño o desventaja en el prójimo y son consecuencias del desarrollo de diferentes factores personales creados en diferentes contextos como familia, colegio, deporte, etc. (Shields \& Bredemeier, 2001). Una importante meta para los investigadores de aspectos morales es determinar los motivos que conducen a los participantes a llevar a cabo acciones antisociales.

En concordancia con lo indicado anteriormente, la teoría del aprendizaje social (Bandura, 1977) define el comportamiento moral como una acción que se ajusta a las normas sociales aprendidas a través de la interacción con los agentes de socialización, en la que existen conceptos adyacentes a los modelos de aprendizaje social, donde los castigos y los refuerzos positivos y negativos se utilizan para enseñar y para modificar los comportamientos.

Por otro lado, y en contraste con la teoría anterior que se centra en que los comportamientos son subyacentes a las normas sociales, la teoría del desarrollo estructural (Haan, 1977, 1991; Kohlberg, 1976, 1981; Piaget, 1932/1965; Rest, 1984) toma en consideración la forma en que el individuo razona y juzga los comportamientos. Así, la moralidad es definida en términos de "juicio sobre conveniencia de determinados comportamientos morales e inmorales" (Bredemeier \& Shields, 1998). La estructura del razonamiento moral, que determina si el comportamiento es correcto o incorrecto, se modifica como consecuencia de los procesos de maduración cognitiva y de la interacción social. Por ello, los individuos son participantes activos en la interpretación de la moralidad a través de la interacción con otros. Según esta teoría, los deportistas son agentes ajenos a los comportamientos morales de 
sus ascendientes, considerando al adolescente como un ente con razonamiento y juicio de valor propio.

Ahora bien, son muchos los estudios que han demostrado la importancia de la familia y, concretamente la influencia de los padres, en el desencadenante de los comportamientos de sus hijos. Según Vazou, Ntoumanis y Duda (2005), la familia se convierte en el núcleo principal donde se producen las primeras experiencias de socialización deportiva donde predomina la prevalencia del comportamiento y adherencia deportiva de los jóvenes, en función de los comportamientos de sus padres.

Centrándonos en ellos, numerosas investigaciones han tratado de explicar la influencia de este colectivo en las posibles conductas de los deportistas. Autores como Stuart y Ebbeck (1995) ponen de manifiesto en sus investigaciones que la implicación hacia el deporte de algunos de los padres parece ser particularmente importante para los niños, especialmente a la hora de llevar a cabo sus decisiones y comportamientos morales en el deporte (Leo, Sánchez-Miguel, Sánchez-Oliva, Amado \& García-Calvo, 2009). Otros estudios, ya marcaban la relevancia del ambiente social de otros significativos como padres, profesores, entrenadores y compañeros en la actuación moral de los deportistas (Shields \& Bredemeier, 2001).

Por otro lado, autores como Genevois (2011) y Wuerth, Lee y Alfermann (2004) destacan la importancia de una atmósfera moral creada por los padres, como posible causa favorecedora o no para la aparición de acciones antideportivas en la práctica de sus hijos. Uno de los trabajos más destacados es el realizado por Guivernau y Duda (2002) en el que encontraron relaciones entre el ambiente moral creado por los padres respecto a la intención y desarrollo de comportamientos antideportivos de los adolescentes, existiendo una relación directa entre los padres y los hijos en la aparición de conductas desadaptativas (Stephens, 2000, 2001). Asimismo, la percepción de los jugadores de un clima que implica al ego creado por los otros significativos se ha identificado con niveles bajos de deportividad (Miller, Roberts \& Ommundsen, 2004; Ommundsen, Roberts, Lemyre \& Treasure, 2003), bajo razonamiento moral (Kavussanu \& Spray,
2006; Ommundsen et al., 2003), la no aceptación de las reglas del juego (Boixadós, Cruz, Torregrosa \& Valiente, 2004; Sánchez-Oliva et al., 2012) y con la mayor intención, juicio y realización de comportamientos antisociales (Kavussanu, 2006; Sage \& Kavussanu, 2008).

Recientemente, autores como Sánchez-Miguel, Leo, Sánchez-Oliva, Amado y García-Calvo (en prensa) en un estudio donde preguntaron a los deportistas por el clima que transmitían sus padres, encontraron respuestas donde manifiestan que la percepción de un clima directivo y de presión por parte de los padres se asocia con una menor satisfacción en la práctica, mientras que la de un clima de apoyo parece favorecer el disfrute de la práctica deportiva de los jóvenes jugadores. Por esto, sería interesante realizar un análisis que centre la atención no solo en los deportistas, sino también en los propios padres, para conocer cuál es el clima motivacional que creen transmitir en sus hijos y si estos determinan la valoración sobre los dilemas morales planteados. Además, este trabajo intenta realizar una comparación entre lo que percibe un deportista y su padre sobre una misma acción de juego en la que se pone de manifiesto la moralidad del individuo.

Así pues, el objetivo principal del estudio se centró en el análisis de las posibles relaciones entre la percepción de comportamientos antisociales de padres e hijos en el contexto deportivo, así como en la valoración de si el clima motivacional que los padres creían trasmitir a sus hijos predecían la aparición de conductas antisociales en los jóvenes. Derivado del objetivo principal, y con base en las investigaciones existentes hasta el momento, se planteó una serie de hipótesis que guiaron esta investigación. La primera hipótesis sugería que las conductas antisociales de los padres se relacionarían positiva y significativamente con los comportamientos antisociales mostrados por los hijos. Como segunda hipótesis se planteó que el fomento de un clima de implicación y ánimo se relacionaría con bajos niveles de comportamientos desadaptativos, mientras que la percepción de un clima de presión y el comportamiento directivo por parte de los padres 
serían predictores de conductas antisociales de los jóvenes deportistas.

\section{Método}

Para el desarrollo de la investigación se ha llevado a cabo un muestreo aleatorio simple en el que ha participado un total de 1420 sujetos, divididos en 710 padres (350 padres y 360 madres), con edades comprendidas entre los 36 y los 49 años $(M=43.56$; $\mathrm{DE}=2.95)$ y sus respectivos hijos/as, 552 de género masculino y 158 de género femenino, con edades comprendidas entre los 11 y los 16 años $(M=12.76$; $\mathrm{DE}=1.15)$, de cuatro deportes colectivos diferentes: fútbol $(\mathrm{N}=453)$, baloncesto $(\mathrm{N}=130)$, balonmano $(\mathrm{N}=65)$ y voleibol $(\mathrm{N}=62)$. Todos ellos de la región de Extremadura, en etapas de formación y que compiten, federativamente, en JUDEX (Juegos Deportivos Extremeños).

Para medir la percepción de los deportistas sobre los comportamientos antisociales, se empleó el factor de actuación, intención antisocial y juicio sobre la acción de la adaptación, del Cuestionario sobre Intenciones y Comportamientos Antisociales en Fútbol ([CICAF]; García-Calvo, 2006). Este instrumento se inicia con una frase introductoria: "A continuación se plantean una serie de escenarios imaginarios que podrían darse en tu deporte y que tienen que ver con comportamientos dentro de una competición. Intenta ponerte en la situación y contestar a las preguntas que le siguen de la forma más sincera posible. No existen respuestas buenas o malas, solo respuestas sinceras". La escala está conformada por siete escenarios antisociales adaptados al colectivo del deportista (p. ej., "Imagina que tu equipo está jugando una final del campeonato y va ganando. Es la última acción del partido y un jugador del equipo contrario puede conseguir éxito y empatar o ganar. Una acción tuya puede evitar el éxito del jugador adversario pero, a la vez, eres consciente de que esa acción (agarrarle o entrarle violentamente por atrás, un bloqueo de forma muy violenta...) puede provocar una lesión grave en el otro jugador") con tres preguntas asociadas a los tres factores, intención antisocial (“iLo harías?"), actuación antisocial ("iHas hecho esto o algo pare- cido alguna vez?”) y juicio sobre la acción (“¿Crees que está bien hacer eso?”), seguido de una escala de 5 puntos. En cuanto a la consistencia interna, se obtuvieron valores de 0.77 para la actuación antisocial, 0.73 para la intención antisocial y 0.8 para el juicio antisocial.

Para evaluar la percepción de los padres sobre los comportamientos antisociales de sus hijos, se empleó el factor actuación, intención antisocial y juicio de la acción de la adaptación del Cuestionario sobre Intenciones y Comportamientos Antisociales en Fútbol ([CICAF]; García-Calvo, 2006). Este instrumento se inicia con una frase introductoria que señala "A continuación, se plantean una serie de escenarios imaginarios que podrían darse en el deporte que practica su hijo/a y que tienen que ver con comportamientos dentro de una competición. Intente ponerse en la situación y contestar a las preguntas de la forma más sincera posible. No existen respuestas buenas o malas, solo respuestas sinceras". Esta herramienta está formada por siete escenarios antisociales (p. ej., "Imagina que el equipo de su hijo/a está jugando una final del campeonato y va ganando. Es la última acción del partido y un jugador del equipo contrario puede conseguir éxito y empatar o ganar. Una acción de su hijo/a puede evitar el éxito del jugador adversario pero, a la vez, eres consciente de que esa acción (agarrarle o entrarle violentamente por atrás, un bloqueo de forma muy violenta...) puede provocar una lesión grave en el otro jugador") acompañado de tres preguntas que corresponden a cada uno de los factores analizados: intención (“iDebería hacerlo?"), actuación (“iTú hijo/a lo ha hecho alguna vez”?) y juicio sobre la acción (¿"Crees que está bien hacer eso?"), con una escala tipo Likert con un rango de 1 a 5 . Los valores de alpha de Cronbach fueron 0.8 para actuación antisocial, 0.76 para intención antisocial y 0.81 para el juicio de la acción.

Se utilizó una versión del instrumento denominado Parental Involvement Sport Questionaire ([PISQ]; Wuerth et al., 2004), empleada en estudios previos realizados con adolescentes por Torregrosa et al. (2007) y validada al castellano por GarcíaCalvo, Leo, Sánchez-Oliva, Amado y Sánchez- 
Miguel (2011). El cuestionario está conformado por un total de 16 ítems, que se agrupan en cuatro factores sobre la percepción que tienen los padres sobre sus hijos en el contexto deportivo. El comportamiento directivo (4 ítems, p. ej.: "le señalo lo que ha hecho mal después de perder una competición"), el comportamiento de apoyo y comprensión (4 ítems, p. ej.: "le felicito por sus esfuerzos después de una competición"), percepción sobre la implicación activa (4 ítems, p. ej.: "ayudo voluntariamente a la organización de las competiciones, ayudo al entrenador, anoto en las competiciones, etc.") y percepción de la presión que le ejercen a sus hijos (4 ítems, p. ej.: "le meto presión para que gane"). Para valorar los comportamientos de los padres, una versión adaptada fue empleada, modificando la frase introductoria original por: "Como padre/ madre, con qué frecuencia adoptas el siguiente comportamiento...". Las posibilidades de respuesta oscilaban en una escala tipo Likert de 5 puntos. En cuanto a la consistencia interna, la escala mostró valores adecuados de alpha de Cronbach de 0.84 para la presión, 0.78 para el comportamiento directivo, 0.72 para la implicación con el club y 0.74 para el apoyo de los padres (Nunnally, 1978).
Para la recogida de datos, se utilizó un protocolo de actuación para hacer equitativa la situación con todos los participantes. En primer lugar, se contactó a las cuatro federaciones deportivas para informarles del objetivo de la investigación. Posteriormente, obtenidos los permisos pertinentes, se les solicitó su voluntaria participación, además de informarlos que sus datos serían tratados confidencialmente. Los datos de los deportistas fueron recogidos en el vestuario antes de un entrenamiento, estando siempre presente el investigador principal para resolver cualquier tipo de duda y cerciorarse de que los formularios se diligenciaran de forma independiente. Una vez cumplido este procedimiento, el investigador les facilitó otro instrumento que debía ser completado por el padre/madre. Finalmente, se requirió que fuera entregado al entrenador/a del equipo al día siguiente.

Para el tratamiento de los datos se utilizó el programa estadístico SPSS 19.0. Asimismo, se comprobó la validez de los cuestionarios (a través de análisis factorial confirmatorio) y la consistencia interna (a través del coeficiente alfa de Cronbach). Seguidamente, se llevó a cavo un análisis descriptivo (media y desviación estándar) de las variables incluidas en el estudio, así como de

TABLA 1

Estadísticos descriptivos y correlaciones entre todas las variables del estudio.

\begin{tabular}{|c|c|c|c|c|c|c|c|c|c|c|}
\hline Variables & 1 & 2 & 3 & 4 & 5 & 6 & 7 & 8 & 9 & 10 \\
\hline 1. Intención AS & - & $0.78 * *$ & $0.43 * *$ & 0.04 & $0.14 * *$ & $0.08 *$ & -0.01 & $0.22 * *$ & $0.28 * *$ & $-0.09 * *$ \\
\hline 2. Actuación AS & & - & $0.41 * *$ & 0.06 & $0.16^{* *}$ & -0.04 & -0.01 & $0.17 * *$ & $0.29 *$ & $0.07 *$ \\
\hline 3. Juicio AS & & & - & $0.22 * *$ & 0.04 & $0.1 * *$ & 0.06 & $0.19 * *$ & $-0.21 * *$ & $-0.26 * *$ \\
\hline 4. Padres Intención AS & & & & - & $0.48 * *$ & $0.34 * *$ & $0.45^{* * *}$ & -0.02 & -0.03 & 0 \\
\hline 5. Padres Actuación AS & & & & & - & 0 & $0.29 * *$ & $0.16^{* *}$ & $0.1 * *$ & -0.06 \\
\hline 6. Padres Juicio AS & & & & & & - & $0.34 * *$ & $0.22 * *$ & $-0.16 * *$ & $-0.16 * *$ \\
\hline 7. Clima directivo & & & & & & & - & -0.06 & -0.03 & -0.02 \\
\hline 8. Clima presión & & & & & & & & - & $0.44 * *$ & $-0.43 * *$ \\
\hline 9. Clima ánimo & & & & & & & & & - & $-0.22 * *$ \\
\hline 10. Clima implicación & & & & & & & & & & - \\
\hline Media & 2 & 1.6 & 1.41 & 1.14 & 1.21 & 1.76 & 2.66 & 1.87 & 4.25 & 2.41 \\
\hline Desviación estándar & 0.92 & 0.77 & 0.91 & 0.31 & 0.39 & 0.72 & 1.05 & 0.97 & 0.68 & 0.74 \\
\hline Asimetría & 0.94 & 1.75 & -1.92 & 3.71 & 2.42 & -4 & 0.39 & 1.19 & -1.22 & 0.55 \\
\hline Kurtosis & 0.39 & 3.42 & 3.32 & 20.28 & 7 & 16.02 & -0.48 & 0.73 & 1.73 & -0.61 \\
\hline alfa de Cronbach & 0.77 & 0.73 & 0.8 & 0.8 & 0.76 & 0.81 & 0.78 & 0.84 & 0.72 & 0.74 \\
\hline
\end{tabular}

$*<0.05 . * *<0.01$.

AS $=$ antisocial.

Fuente: elaboración propia. 
Pedro Antonio Sánchez Miguel, Juan José Pulido González, Diana Amado Alonso, David Sánchez Oliva, Francisco Miguel Leo Marcos

TABLA 2

Coeficientes del análisis de regresión lineal considerando como variable dependiente la intención antisocial de los deportistas

\begin{tabular}{|c|c|c|c|c|}
\hline Variable & $\beta$ & $R^{2}$ & $t$ & $p$ \\
\hline & & 0.1 & & \\
\hline Padres clima directivo & 0.02 & & 0.63 & 0.52 \\
\hline Padres clima presión & 0.09 & & 2.34 & 0.01 \\
\hline Padres clima ánimo & -0.02 & & -0.67 & 0.5 \\
\hline Padres clima implicación & -0.02 & & -0.68 & 0.49 \\
\hline Padres intención AS & 0.1 & & 2.59 & 0.01 \\
\hline Padres actuación AS & 0.21 & & 5.48 & 0 \\
\hline Padres juicio AS & 0.01 & & 2.31 & 0.01 \\
\hline
\end{tabular}

AS $=$ antisocial.

Fuente: elaboración propia.

correlaciones bivariadas mediante el coeficiente de correlación de Pearson.

\section{Resultados}

En la Tabla 1 se muestran los resultados descriptivos y correlaciones obtenidas de las diferentes variables del estudio. Referente a los descriptivos, se pueden observar valores medios discretos en las variables referentes a comportamientos desadaptativos (intención, actuación y juicio antisocial), tanto en los padres como en los deportistas. Seguidamente, teniendo en cuenta el clima que creen transmitir los padres a sus hijos, destaca el clima de ánimo, mientras que los climas directivos y de implicación, obtienen valores por encima del clima de presión. Finalmente, tras observar los valores de la asimetría y curtosis, se aprecia que los datos se reúnen de acuerdo con la frecuencia que se halla dentro de la distribución, y determinando un alto grado de concentración respecto a ella.

En las correlaciones bivariadas, destacan las relaciones halladas entre los comportamientos antisociales de los padres asociados con los de sus hijos. La intención antisocial del deportista se relaciona positivamente con la actuación antisocial que perciben de sus padres, como también con el clima de presión. Por otro lado, la percepción de actuación antisocial del deportista se relaciona de forma positiva con el clima de presión y con la actuación antisocial de los padres. Por último, el juicio antisocial de los adolescentes se relaciona negativamente con el clima de ánimo y con el clima de implicación y de forma positiva con el juicio e intención antisocial de los padres.

TABLA 3

Coeficientes del análisis de regresión lineal considerando como variable dependiente la actuación antisocial de los deportistas

\begin{tabular}{|c|c|c|c|c|}
\hline Variable & $\beta$ & $R^{2}$ & $t$ & $p$ \\
\hline & & 0.1 & & \\
\hline Padres clima directivo & 0.02 & & 0.5 & 0.61 \\
\hline Padres clima presión & 0.14 & & 3.48 & 0 \\
\hline Padres clima ánimo & 0.02 & & 0.59 & 0.55 \\
\hline Padres clima implicación & -0.06 & & -0.61 & 0.1 \\
\hline Padres intención AS & 0.02 & & 0.69 & 0.48 \\
\hline Padres actuación AS & 0.26 & & 6.75 & 0 \\
\hline Padres juicio AS & 0 & & 2.58 & 0.01 \\
\hline
\end{tabular}

AS $=$ antisocial

Fuente: elaboración propia. 
En la Tabla 2 se representa el análisis de regresión lineal por introducir, incluyendo como posibles consecuencias las percepciones de comportamiento de los deportistas y como posibles variables predictoras todas las relacionadas con el colectivo de padres. En primer lugar, tal y como se aprecia en la Tabla 2, las variables que predicen de forma significativa la intención social de los deportistas son, por un lado, el clima de presión que creen transmitir los padres y, por otro, la intención, actuación y juicio antisocial de los mismos, con una varianza explicada aceptable.

En la Tabla 3 se toma como variable dependiente la percepción de actuación social de los deportistas. Para ello, las posibles variables predictoras han sido todas las asociadas con los padres de los deportistas. Los resultados muestran que los mayores predictores de esta conducta desadaptativa son el clima de presión que cree ejercer el padre sobre su hijo, así como la propia percepción antisocial que enjuicia el padre. Sin embargo, no se encuentran predicciones de las variables de forma negativa como el clima de ánimo o el de implicación. Las variables de clima directivo y la intención antisocial de los padres no predice de forma positiva la variable dependiente considerada. La varianza explicada es adecuada por encima del $10 \%$.

En la Tabla 4 aparecen los datos referentes al juicio antisocial de los deportistas, donde la intención, la actuación antisocial y el juicio de los padres se muestran como los mayores predictores de esta conducta desadaptativa, con una varianza adecuada del $16 \%$. Por el contrario, se aprecia que ninguna de las variables del clima motivacional promovido por los padres, emergen como predictores del juicio antisocial de los participantes en deportes colectivos.

\section{Discusión}

El objetivo del presente estudio fue analizar las posibles relaciones entre la percepción de comportamientos antisociales de padres e hijos en cuatro modalidades deportivas de equipo diferentes y valorar si el clima motivacional que los padres creían transmitir a sus hijos guardaba alguna relación con estas conductas desadaptativas por parte de los deportistas.

En primer lugar, se planteó como hipótesis que las percepciones antisociales de los padres se relacionarían de forma positiva con las percepciones antisociales de sus hijos. En este sentido, tanto la intención antisocial, la actuación y el juicio emitido del escenario planteado se relaciona positivamente con las percepciones encontradas en los deportistas. Por lo tanto, se puede indicar que existe una prevalencia significativa en los pensamientos de los padres y los adolescentes como habían adelantado Guivernau y Duda (2002), en el que el ambiente creado por los significativos del deporte, en este caso los padres, guarda una estrecha relación con la intención y desarrollo de comportamientos desadaptativos de los deportistas. Además, si consideramos el fuerte protagonismo que ejerce la familia

TABLA 4

Coeficientes del análisis de regresión lineal considerando como variable dependiente el juicio antisocial de los deportistas

\begin{tabular}{|c|c|c|c|c|}
\hline Variable & $\beta$ & $R^{2}$ & $t$ & $p$ \\
\hline Modelo & & 0.16 & & \\
\hline Padres clima directivo & -0.01 & & -0.21 & 0.83 \\
\hline Padres clima presión & -0.01 & & -0.33 & 0.74 \\
\hline Padres clima ánimo & 0.03 & & 0.9 & 0.36 \\
\hline Padres clima implicación & 0.04 & & 1.02 & 0.3 \\
\hline Padres intención AS & 0.11 & & -2.83 & 0 \\
\hline Padres actuación AS & 0.14 & & -3.66 & 0 \\
\hline Padres juicio AS & 0.27 & & 5.4 & 0 \\
\hline
\end{tabular}

$\mathrm{AS}=$ antisocial.

Fuente: elaboración propia. 
en estas edades (Vazou et al., 2005), los comportamientos de los jóvenes pueden considerarse como meras reproducciones de la atmósfera creada en el seno familiar. Este hallazgo también es consistente con los encontrados por Sánchez-Miguel et al. (en prensa) quiénes demostraron que la percepción de los comportamientos desadaptativos de los padres por parte de los deportistas estaba relacionado con el aumento de conductas negativas en el contexto deportivo. Por ello, estos resultados demuestran la gran importancia que adquieren en los procesos de socialización en edades tempranas los padres de los jóvenes deportistas, demostrando cómo aspectos como el apoyo emocional, información y oportunidades están relacionados con la cantidad y calidad de la práctica deportiva (Jowett \& Timson-Katchis, 2005; Weiss \& Fretwell, 2005). Además, teniendo en cuenta la teoría del aprendizaje social (Bandura, 1977), podría estar relacionado con el hecho de que los comportamientos de los deportistas vienen muy influenciados por los agentes de socialización, en este caso por los padres. De esta manera, se puede decir que la primera hipótesis planteada se cumple.

Seguidamente, la segunda hipótesis indicaba que una percepción de los padres en transmitir un clima de ánimo e implicación a sus hijos prediría niveles bajos de comportamientos desadaptativos, mientras que un clima de presión y comportamiento directivo emergerían como predictores de altos comportamientos desadaptativos. Tras observar los resultados, se puede afirmar que no existe ninguna predicción entre el clima creado por los padres de ánimo e implicación con la emisión de una respuesta no antideportiva de sus hijos. Además, nuestros resultados indican que tampoco eran predictores del juicio antisocial valorado por el joven deportista. Estos resultados no son consistentes con los hallados por autores como Genevois (2011) y Wuerth et al. (2004) quiénes dieron constancia de la importancia de la atmósfera moral creada por los padres en la aparición de situaciones favorecedoras o no de comportamientos antideportivos. La teoría del desarrollo estructural (Haan, 1977, 1991; Kohlberg, 1976, 1981; Piaget, 1932/1965; Rest, 1984) defiende que el individuo razona y juzga los comportamientos y que estos no vienen subordinados por ningún agente socializador, así pues, el deportista no estaría influenciado por el clima motivacional creado por su padre, y sería un ente independiente con juicio de valor propio. En relación con esto, Sánchez-Miguel et al. (en prensa) reveló que un clima de ánimo e implicación y un apoyo por parte de los padres percibido por los deportistas, conlleva a una menor tasa de abandono deportivo de manera significativa. Una posible explicación es que el cuestionario lo rellenó el padre/madre que estaba más implicado en la práctica deportiva, valorando conductas adaptativas, por lo cual es más probable que presente mayores comportamientos adaptativos, aunque el entorno completo (padre y madre) influya finalmente en que no surja ningún tipo de predicción significativa.

Por otro lado, se obtuvo que un clima de presión creado por los padres fue el mayor predictor para que los hijos respondiesen a los escenarios planteados en las variables de actuación e intención antisocial, desde un punto de vista antideportivo. Sin embargo, un clima directivo no predice ni la actuación ni la intención antisocial de los deportistas. Estudios similares como el de Miller et al. (2004) hallaron que un clima que valoraba principalmente el resultado y no el esfuerzo en los padres se relacionaba positivamente con bajos niveles de deportividad, así como con la no aceptación de las reglas de juego (Boixadós et al., 2004), muy relacionada con la emisión de un juicio y la intención y actuación de una situación moral planteada. Asimismo, en un trabajo similar con escenarios creados para poner de manifiesto los dilemas morales de los adolescentes (Leo, Sánchez-Miguel, Sánchez-Oliva, Amado et al., 2009; Leo, Sánchez-Miguel, Sánchez-Oliva, Gómez et al., 2009) hallaron que un clima que favorecía la superación y se valoraba a los deportistas en función de un criterio autorreferencial, estaba relacionado con comportamientos adaptativos sociales. Además, en relación con esta segunda hipótesis, se destaca que el clima de presión emergía como el mayor predictor de los comportamientos desadaptativos en el contexto deportivo. Sin embargo, el estado de ánimo e implicación no era precedido por bajos comportamientos antisociales. 
Así pues, la principal conclusión que se puede extraer del presente trabajo es la relación entre las percepciones de comportamientos antideportivos mostrados por los padres con las percepciones de sus hijos deportistas. Además, el clima de presión de los padres es un predictor significativo de actuaciones e intenciones antisociales de sus hijos durante la práctica deportiva. Por este motivo, los padres se muestran cruciales en la aparición y/o aceptación de comportamientos antisociales. Diferentes estrategias como las empleadas por García-Calvo et al. (2012), basadas en el aprendizaje de valores positivos, educación integral en el entorno familiar, etc., pueden ser empleadas para conseguir una atmósfera moral adecuada en el entorno próximo del joven deportista.

En cuanto a las limitaciones del estudio, hay que señalar que solamente se valora la percepción del padre/madre más implicado con la práctica deportiva de su hijo/a, siendo necesario valorar a todos los padres/madres de deportistas ya que son generadores del ambiente moral que se desarrolla en edades tempranas. En segundo lugar, este estudio valora la percepción de comportamientos antisociales, no se miden directamente las conductas y comportamientos antisociales que se producen en el contexto deportivo, lo cual puede ofrecer información complementaria a la obtenida en este estudio junto con la valoración directa de los comportamientos antisociales de los padres en el contexto deportivo.

Para finalizar, y en relación con lo indicado previamente, este estudio aporta algunas prospectivas de futuro que se deben trabajar en el deporte escolar. La valoración directa mediante la conducta verbal de los padres en entrenamientos y competición arrojaría importante información sobre la importancia de este significativo en la aceptación o rechazo de comportamientos antisociales. Asimismo, la inclusión de nuevos significativos como entrenadores (Balaguer, Castillo \& Duda, 2008) de clubes de fútbol, sería un aspecto fundamental para analizar en el contexto escolar.

\section{Referencias}

Abenza, L., Bravo, J. F. \& Olmedilla, A. (2006). Estrategias psicológicas para una intervención en crisis: un caso en balonmano femenino. Revista de Psicología del Deporte, 15 (1),109-125.

Balaguer, I., Castillo, I. \& Duda, J. L. (2008). Apoyo a la autonomía, satisfacción de las necesidades, motivación y bienestar en deportistas de competición: un análisis de la teoría de la autodeterminación. Revista de Psicología del Deporte, 17 (1), 123-139.

Bandura, A. (1991). Social cognitive theory of moral thought and action. En W.M. Kurtines \& J.L. Gewirtz (Eds.), Handbook of moral behavior and development: Theory, research, and applications (1), 71-129. Hillsdale, NJ: Erlbaum.

Bandura, A. (1977). Social learning theory. Englewood Cliffs, NJ: Prentice-Hall.

Boixadós, M., Cruz, J., Torregrosa, M. \& Valiente, L. (2004). Relationship among motivational climate, satisfaction, perceived ability and fair play attitudes in young soccer players. Journal of Applied Sport Psychology, 16 (4), 301-317.

Bredemeier, B. J. L. \& Shields, D. L. (1998). Moral in assessment in sport psychology. En J. L. Duda (Ed.), (pp. 257-276). Morgantown, WV: Fitness Information Technology.

García-Calvo, T. (2006). Motivación y comportamientos adaptativos en jóvenes futbolistas. Tesis doctoral, Universidad de Extremadura, España.

García-Calvo, T., Leo, F. M., Sánchez-Oliva, D., Amado, D. \& Sánchez-Miguel, P.A. (2011, julio). Validation of the Parental Involvement in Sport Questionnaire in Spanish Population. Comunicación presentada en el XIII Congreso de Psicología del Deporte, Madeira, Portugal.

García-Calvo, T., Sánchez-Oliva, D., Sánchez-Miguel, P. A., Leo, F. M. \& Amado, D. (2012). Escuela del deporte: valoración de una campaña para la promoción de valores. Motricidad, European Journal of Human Movement, 28 (2), 12-18.

Genevois, P. C. (2011). El rol de los padres en el entrenamiento de los tenistas iniciantes. ITF Coaching and Sport Science Review, 55 (19), 27-28. 
Guivernau, M. \& Duda, J. L. (2002). Moral atmosphere and athletic aggressive tendencies in young soccer players. Journal of Moral Education, 31 (1), 67-85.

Haan, N. (1977). Coping and defending: Processes of selfenvironment organization. New York: Academic Press.

Haan, N. (1991). Moral development and action from a social constructivist perspective. En W. M. Kurtines \& J. L. Gerwirtz (Eds.), Handbook of moral behavior and development, Vol. 1: Theory (Vol. 1, pp. 251-273). Hillsdale, NJ: Erlbaum.

Jowett, S. \& Timson-Katchis, M. (2005). Social networks in sport: Parental influence on the coachathlete relationship. Sport Psychologist, 19 (3), 267-287.

Kavussanu, M. (2007). Morality in sport. En S. Jowett \& D. E. Lavallee (Eds.), Social Psychology in Sport, (pp. 265-278). Champaign IL: Human Kinetics.

Kavussanu, M. \& Boardley, I. D. (2009). The prosocial and antisocial behavior in Sport Scale. Journal of Sport y Exercise Psychology, 31, (1), 97-117.

Kavussanu, M. \& Spray, C. M. (2006). Contextual influences on moral functioning of male youth footballers. Sport Psychologist, 20 (1), 1-23.

Kavussanu, M., Seal, A. R. \& Phillips, D. R. (2006). Observed prosocial and antisocial behaviors in male soccer teams: Age differences across adolescence and the role of motivational variables. Journal of Applied Sport Psychology, 18 (4), 326-344.

Kohlberg, L. (1976). Moral stages and moralization: The cognitive-developmental approach. En T. Lickona (Ed.), Moral development and behaviour: Theory, research, and social issues (pp. 31-53). New York: Holt, Rineheart \& Winston.

Kohlberg, L. (1981). Essays on moral development: Vol. 1. The philosophy of moral development. (Vol. 1) San Francisco: Harper \& Row.

Lee, M. J., Whitehead, J. \& Balchin, N. (2000). The measurement of values in youth sport: Development of the Youth Sport Values Questionnaire. Journal of sport and exercise psychology, 22 (4), 307-326.

Lee, M. J., Whitehead, J., Ntoumanis, N. \& Hatzigerorgiadis, A. (2008). Relationships among values, achievement orientations, and attitudes in youth sport. Journal of sport and exercise psychology, 30 (5), 588-610.

Leo, F. M., Sánchez-Miguel, P. A., Sánchez-Oliva, D., Gómez, F. R. \& García-Calvo, T. (2009). Análisis de las relaciones existentes entre la orientación y el clima motivacional con los comportamientos antisociales en jóvenes deportistas. Revista Iberoamericana de Psicología del Ejercicio y del Deporte, 4 (1), 15-28.

Leo, F. M., Sánchez-Miguel, P. A., Sánchez-Oliva, D., Amado, D. \& García-Calvo, T. (2009). Interacción entre la percepción del comportamiento de los padres y los comportamientos deportivos en edades de iniciación. Acción psicológica, 6 (2), 55-62.

Luckwü, R. M. \& Guzmán, J. F. (2011). Deportividad en balonmano: un análisis desde la Teoría de la $\mathrm{Au}$ todeterminación. Revista de Psicología del Deporte, 20 (2), 305-320.

Miller, B. W., Roberts, G. C. \& Ommundsen, Y. (2004). Effect of motivational climate on sportspersonship among competitive youth male and female football players. Scandinavian Journal of Medicine and Science in Sports, 14, (3), 193-202.

Nunnally, J. C. (1978). Psychometric theory. New York: McGraw-Hill.

Ommundsen, Y., Roberts, G. C., Lemyre, P.N. \& Treasure, D. (2003). Perceived motivational climate in male youth soccer: Relations to social-moral functioning, sportspersonship and team norm perceptions. Psychology of Sport and Exercise, 4 (4), 397-413.

Piaget, J. (1965). Moral judgment of the child (M. Gabain, Trad.). New York: Free Press. (Trabajo original publicado en 1932)

Rest, J. R. (1984). The major component of morality. En W. Kurtines \& J. Gerwirtz (Eds.), Morality, moral behavior and moral development (pp. 24-40). New York: Wiley.

Sage, L. (2006). Predictors of moral behaviour in football. Tesis Doctoral, University of Birmingham, Reino Unido.

Sage, L. \& Kavussanu, M. (2008). Goal orientations, motivational climate, and prosocial and antisocial behaviour in youth football: Exploring their temporal stability and reciprocal relationships. Journal of Sports Sciences, 26 (7), 717-732. 
Sánchez-Miguel, P. A., Leo, P. A., Sánchez-Oliva, D., Amado, D. \& García-Calvo, T. (en prensa-a). The importance of parents' behavior in their children's enjoyment and amotivation in sports. Journal of Human Kinetics.

Sánchez-Miguel, P. A., Leo, P. A., Sánchez-Oliva, D., Amado, D. \& García-Calvo, T. (en prensa-b). La influencia del entorno próximo sobre la persistencia en la práctica de actividad físico-deportiva. Cuadernos de psicología del deporte.

Sánchez-Oliva, D., Leo, F. M., Sánchez-Miguel, P. A., Amado, D. \& García-Calvo, T. (2012). Antecedentes motivacionales de los comportamientos prosociales y antisociales en el contexto deportivo. Revista Internacional de Medicina y Ciencias de la Actividad Física y del Deporte, 12 (46), 253-270.

Shields, D. L. \& Bredemeier, B. J. L. (2001). Moral development and behavior in sport. En R. N. Singer, H. A. Hausenblas \& C. M. Janelle (Eds.), Handbook of sport psychology, (pp. 585-603). New York: John Wiley \& Sons.

Stephens, D. E. (2000). Predictors of likelihood to aggress in youth soccer: An examination of coed and all-girls teams. Journal of Sport Behavior, 23 (3), 311-325.

Stephens, D. E. (2001). Predictors of aggressive tendencies in girls' basketball: An examination of begin- ning and advanced participants in a summer skills camp. Research Quarterly for Exercise and Sport, 72 (3), 257-266.

Stuart, M. E. \& Ebbeck, V. (1995). The influence of perceived social approval on moral development in youth sport. Pediatric Exercise Science, 7 (3), 270-280.

Torregrosa, M., Cruz, J., Sousa, C., Vildarich, C., Villamarín, F., García-Mas, A., et al. (2007). La influencia de padres y madres en el compromiso deportivo de futbolistas jóvenes. Revista Latinoamericana de Psicología, 39, (2), 227-237.

Vallerand, R. J. (2007). Intrinsic and extrinsic motivation in sport and physical activity. En G. Tenenbaum \& R. C. Eklund (Eds.), Handbook of sport psychology, (pp. 59-83). New York: Wiley.

Vazou, S., Ntoumanis, N. \& Duda, J. L. (2005). Peer motivational climate in youth sport: A qualitative inquiry. Psychology of Sport and Exercise, 6 (5), 497-516.

Weiss, M. R. \& Fretwell, S. D. (2005). The parent-coach/ child-athlete relationship in youth sport: Cordial, contentious, or conundrum? Research Quarterly for Exercise and Sport, 76 (3), 286-305.

Wuerth, S., Lee, M. \& Alfermann, D. (2004). Parental involvement and athletes' career in youth sport. Psychology of Sport and Exercise, 5 (1), 21-33. 
